\title{
Differences in content and organisational aspects of pulmonary rehabilitation programmes
}

\author{
Martijn A. Spruit ${ }^{1}$, Fabio Pitta ${ }^{1}$, Chris Garvey, Richard L. ZuWallack, \\ C. Michael Roberts, Eileen G. Collins, Roger Goldstein, Renae McNamara, \\ Pascale Surpas, Kawagoshi Atsuyoshi, José-Luis López-Campos, \\ Ioannis Vogiatzis, Johanna E.A. Williams, Suzanne Lareau, Dina Brooks, \\ Thierry Troosters, Sally J. Singh, Sylvia Hartl, Enrico M. Clini, and \\ Emiel F.M. Wouters, on behalf of the ERS Rehabilitation and Chronic Care, and \\ Physiotherapists Scientific Groups, the American Association of Cardiovascular \\ and Pulmonary Rehabilitation, the ATS Pulmonary Rehabilitation Assembly and \\ the ERS COPD Audit team
}

Affiliations: For a full list of the authors affiliations please see the Acknowledgements section. ${ }^{1}$ Both authors contributed equally.

Correspondence: M.A. Spruit, Dept of Research and Education, Centre of Expertise for Chronic Organ Failure (CIRO+], Hornerheide 1, 6085 NM, Horn, the Netherlands. E-mail: martijnspruit@ciro-horn.nl

ABSTRACT The aim was to study the overall content and organisational aspects of pulmonary rehabilitation programmes from a global perspective in order to get an initial appraisal on the degree of heterogeneity worldwide.

A 12-question survey on content and organisational aspects was completed by representatives of pulmonary rehabilitation programmes that had previously participated in the European Respiratory Society (ERS) COPD Audit. Moreover, all ERS members affiliated with the ERS Rehabilitation and Chronic Care and/or Physiotherapists Scientific Groups, all members of the American Association of Cardiovascular and Pulmonary Rehabilitation, and all American Thoracic Society Pulmonary Rehabilitation Assembly members were asked to complete the survey via multiple e-mailings.

The survey has been completed by representatives of 430 centres from 40 countries. The findings demonstrate large differences among pulmonary rehabilitation programmes across continents for all aspects that were surveyed, including the setting, the case mix of individuals with a chronic respiratory disease, composition of the pulmonary rehabilitation team, completion rates, methods of referral and types of reimbursement.

The current findings stress the importance of future development of processes and performance metrics to monitor pulmonary rehabilitation programmes, to be able to start international benchmarking, and to provide recommendations for international standards based on evidence and best practice.

@ERSpublications

Differences in aspects of pulmonary rehabilitation programmes suggest caution in generalisation of research findings http://ow.ly/qOJhl

For editorial comments see page 1223.

This article has supplementary material available from www.erj.ersjournals.com

Received: Aug 192013 | Accepted after revision: Nov 012013 | First published online: Dec 122013

Support statement: This study was partially funded by the Weijerhorst Foundation (Maastricht, the Netherlands).

Conflict of interest: None declared.

Copyright @ERS 2014 


\section{Introduction}

Pulmonary rehabilitation is defined as a comprehensive intervention based on thorough patient assessment followed by patient-tailored therapies that include, but are not limited to, exercise training, education and behaviour change. These are designed to improve the physical and psychological condition of people with chronic respiratory disease and to promote the long-term adherence of health-enhancing behaviours [1]. Pulmonary rehabilitation has consistently shown that individuals with chronic respiratory disease experience: a decrease in daily symptoms of dyspnoea, fatigue, anxiety and depression; improvements in exercise performance, self-efficacy and health status; and a decrease in healthcare utilisation [2-9]. As pulmonary rehabilitation addresses the symptoms, activity limitations and reduced health-related quality of life associated with chronic respiratory disease, it is now considered a fundamental component of the integrated disease management of individuals with chronic respiratory disease [10].

Although pulmonary rehabilitation should be made available to all individuals with chronic respiratory disease who still suffer from daily symptoms despite optimal medical therapy [1], there are still marked shortfalls in the provision of this intervention [11-15]. Moreover, there is variability in content and organisational aspects among pulmonary rehabilitation programmes at a national level [11, 14-17]. This is most probably the result of local conditions, and not a reflection of an evidence-based organisational model. Differences in content and organisational aspects may partially explain the differences in reported outcomes following pulmonary rehabilitation [18].

To date, neither the content nor the organisational aspects of pulmonary rehabilitation programmes have been studied worldwide during the same period of time. As international differences may limit international benchmarking [19], the overall content and organisational aspects of pulmonary rehabilitation programmes from a global perspective were studied to get an initial appraisal on the degree of heterogeneity worldwide.

\section{Methods}

\section{Study design}

An existing survey on pulmonary rehabilitation was made available for this project by BrooKs and coworkers $[11,16]$. The survey was shortened to 12 questions. The response format was either a checkbox or free text option (see online supplementary material). From September 2012 to February 2013, pulmonary rehabilitation programmes that previously participated in the European Respiratory Society (ERS) COPD Audit, were asked to complete this survey $(n=384)$. Moreover, all ERS members affiliated with the ERS Rehabilitation and Chronic Care and/or Physiotherapists Scientific Groups $(n=983)$, all members of the American Association of Cardiovascular and Pulmonary Rehabilitation (AACVPR, $\mathrm{n}=1628$ ), and all American Thoracic Society (ATS) Pulmonary Rehabilitation Assembly members $(n=486)$ were asked to complete the survey via multiple e-mailings. There may have been overlap as people can be affiliated to two or more of the abovementioned groups. Moreover, not all recipients of the e-mail work in a pulmonary rehabilitation setting. Surveys were returned by e-mail or post. All data were inserted into an Excel (Microsoft Corporation, Redmond, WA, USA) database. Respondents were contacted via e-mail if data were missing. Pulmonary rehabilitation programmes starting after 2011 were excluded. Only complete surveys were used for further analyses. Only one survey per pulmonary rehabilitation programme was included in the analyses (i.e. only the first completed received survey per pulmonary rehabilitation programme was used).

\section{Statistical analyses}

GraphPad Prism 5 (GraphPad Software Inc., La Jolla, CA, USA), Excel (Microsoft Corporation) and SPSS (SPSS Inc., Chicago, IL, USA) were used for the statistical analyses. Median (interquartile range (IQR)) and proportion were calculated as appropriate. A posteriori differences between Europe and North America were analysed using a Wilcoxon rank-sum test or Chi-squared test as these were the two continents with the highest number of completed surveys. The findings of the remaining continents were not clustered as the number of surveys was rather limited. A priori the level of significance was set at $p \leqslant 0.05$.

\section{Results}

General characteristics

A total of 481 surveys were returned from 40 countries. After excluding all surveys with missing data or overlapping pulmonary rehabilitation programmes, 430 surveys were available for the final analyses. Most surveys were affiliated with pulmonary rehabilitation programmes from Europe (43.7\%) or North America (43.5\%). The remaining programmes were from Oceania, Asia, South America or Africa (fig. E1). 


\section{Setting}

Most commonly, centres offered structured outpatient pulmonary rehabilitation programmes (262 $(60.9 \%)$ centres), 41 (9.5\%) centres offered inpatient programmes, and $106(24.7 \%)$ centres offered both. The remaining $21(4.9 \%)$ pulmonary rehabilitation programmes offered rehabilitation in the home setting (including tele-rehabilitation) or the primary care setting. Most of these programmes (17 of 21) were offered in combination with an inpatient or outpatient hospital-based pulmonary rehabilitation programme. Most programmes in Europe and North America were outpatient programmes (table 1).

\section{Types of reimbursement}

A small number (six $(1.4 \%)$ ) of programmes did not receive any reimbursement for providing pulmonary rehabilitation. The remaining pulmonary rehabilitation programmes were funded by one or more sources (range: 1-5): individuals' insurance (58.8\%), government (54.8\%), individuals' own money (25.6\%), workplace insurance $(10.2 \%)$, or others including, but not limited to, departmental funding, research grants, external funding, personal efforts and private donations. Programmes were more likely to be reimbursed in Europe by the government, while in North America the programmes were more likely to be reimbursed by the patient's insurance or own money (table 1).

\section{Team members}

Pulmonary rehabilitation teams consisted of a median (IQR) of 5 (4-7) healthcare professionals (fig. 1a). Chest physicians $(70.7 \%)$, dieticians $(70.2 \%)$, nurses $(66.7 \%)$ and physiotherapists $(61.4 \%)$ were the most prevalent team members (fig. 1b). Chest physicians, physiotherapists, occupational therapists, social workers, psychologists and cardiologists were more frequent participants in Europe than North America. In North America, dieticians, exercise physiologists and respiratory therapists were more common (table 1).

\section{Referral sources}

Individuals with chronic respiratory disease were referred by a median (IQR) of 2 (2-3) referral sources. Chest physicians and general practitioners referred individuals with chronic respiratory disease to $95.3 \%$ and $73.3 \%$ of the pulmonary rehabilitation programmes, respectively. Interestingly, self-referral was possible in $29.8 \%$ of the pulmonary rehabilitation programmes (fig. 2). It was more common for programmes in North America to report referrals from general practitioners, self-referrals or cardiologists than in Europe (table 1).

\section{Patient volumes and completion rates}

The respondents estimated that a total of 34890 individuals with chronic respiratory disease enrolled in pulmonary rehabilitation programmes in 2011 (median 40 to 75 enrolled individuals per programme) (fig. 3a). The median proportion of individuals with a chronic respiratory disease that completed a pulmonary rehabilitation programme in 2011 was estimated to be $75-90 \%$ (fig. 3b). More patients were enrolled from Europe in 2011 than North America (table 1).

\section{Case mix}

The case mix of individuals with chronic respiratory disease consisted of a median (IQR) of 6 (4-7) different primary diagnoses (fig. 4a). Most pulmonary rehabilitation programmes received referrals of individuals with "stable" chronic obstructive pulmonary disease (COPD) (97.4\%), COPD during and/or directly following an exacerbation $(74.9 \%)$, restrictive lung disease (including interstitial lung disease and thoracic wall diseases; 73.7\%), asthma (71.9\%) or post-thoracic surgery (71.9\%) (fig. 4b). Programmes from North America reported more frequent referrals for restrictive lung disease, asthma and post-thoracic surgery than those from Europe (table 1).

\section{Duration and frequency}

The median (IQR) length of the pulmonary rehabilitation programmes was 9 (8-12) weeks. Individuals with a chronic respiratory disease attended a median (IQR) of 2.5 (2-3) pulmonary rehabilitation sessions per week, for a median (IQR) duration of 1.5 (1-2) h per session. The median (IQR) number of hours of pulmonary rehabilitation dosage (number of weeks $\times$ number of sessions per week $\times$ number of hours per session) was similar for government funded programmes (32 (24-48) h), insurance funded programmes (36 (27-54) h), or a combination thereof $(36(27-48) h ; p=0.31)$. Programmes in North America were longer in duration compared to European programmes, while the number of sessions per week was greater and the duration of sessions was longer for European programmes. In turn, the total median hours of pulmonary rehabilitation dosage provided in Europe or North America was similar (table 1). 
TABLE 1 European versus North American pulmonary rehabilitation programmes

\section{Europe}

Subjects n

Setting

Outpatient

Inpatient

Both

Other

Types of reimbursement ${ }^{\#}$

Own insurance

Government

Own money

Work insurance

Not funded

Other

Pulmonary rehabilitation team members"

Chest physician

Physiotherapist

Occupational therapist

Social worker

Psychologist

Dietician

Exercise physiologist

Internist

Cardiologist

General practitioner

Pharmacist

Nurse

Respiratory therapist

Number of team members

Referral sites"

Chest physicians

General practitioners

Self-referral

Specialist respiratory nurse

Cardiologist

Median number of individuals per pulmonary rehabilitation programme ${ }^{+}$

Estimated total pulmonary rehabilitation enrolments in $2011 \mathrm{n}$

Median percentage of pulmonary rehabilitation completers

Case mix $^{\#}$

Stable COPD

Unstable COPD

Restrictive lung disease $\mathrm{e}^{\S}$

Asthma

Post-thoracic surgery

Length of pulmonary rehabilitation programme weeks

Number of pulmonary rehabilitation sessions per week

Duration of pulmonary rehabilitation session $h$

Total pulmonary rehabilitation $\mathrm{h}^{f}$

Most important outcomes"

Quality of life

6-min walk test

Dyspnoea

Depression

Physical activity

Self-management skills

Smoking cessation

Activities of daily living

Interventions

Outdoor walking

Treadmill walking

Stationary cycling

Resistance training using training apparatus

Resistance training using handheld weights

Education

ECT/ADL training

Self-management training

Nutritional support

Inspiratory muscle training

Neuromuscular electrical stimulation

Breathing exercise including PLB

Smoking cessation

Psychosocial support

Number of types of interventions
188

48.9

16.0

29.3

5.8

38.3

62.8

10.6

3.7

2.7

87.8

95.2

36.2

42.6
53.7

76.1
25.5
11.7

11.7

43.0

18.1

22.9

68.1

1.6
$6(4-8)$

96.8

55.9

21.3

12.2

1.1
76 to 105

19515

76 to 90

95.7
74.5
66.5
63.3
61.7
$8(5.6-12)$
$2.5(2-4)$
$2(1.3-2)$
$39.3(25.0-62.5$

81.4
33.0
48.4
3.2
33.5
33.5
5.9
28.7

43.6
68.6
89.4
62.8
71.3
89.9
64.9
75.5
76.1
54.8
29.3
86.7
76.6
64.9

10 (8-12)
North America

187

71.7

3.7

1.6

92.5

38.5

43.3

19.3
0.5

0.5

62.0

17.1
17.1

17.1
24.1

8.6

93.5

62.6
8.0

20.0

13.4

18.2

64.2

59.9
$4(3-6)$

95.7

92.5

33.7

3.7

9.1

46 to 75

11475

61 to 75

$\begin{array}{ll}98.9 & 0.11\end{array}$

$\begin{array}{ll}75.9 & 0.81\end{array}$

$82.4 \quad 0.001$

$82.9<0.001$

$11(8-12)$

$2.5(2-3)$

$1.5(1-1.5)$

$36(30-48)$

$<0.001$
$<0.001$

0.005

$<0.00$

0.33

$\begin{array}{lc}83.4 & 0.68 \\ 53.5 & <0.001\end{array}$

$\begin{array}{lr}53.5 & <0.001 \\ 35.8 & 0.016\end{array}$

$\begin{array}{ll}9.1 & 0.019\end{array}$

$\begin{array}{ll}21.9 & 0.02\end{array}$

$30.5-0.58$

$\begin{array}{cc}16.0 & 0.002 \\ 36.4 & 0.12\end{array}$

5.9

$\begin{array}{ll}5.9 & <0.001 \\ 97.9 & <0.001\end{array}$

$\begin{array}{ll}97.3 & 0.003 \\ 67.9 & 0.33\end{array}$

$97.9-0.33$
$93.6-0.001$

$93.6<0.001$

$\begin{array}{ll}98.9 & <0.001 \\ 94.7 & <0.001\end{array}$

$85.0<0.001$

$93.6<0.001$

$\begin{array}{ll}93.6 & 0.35\end{array}$

$\begin{array}{ll}1.6 & <0.001\end{array}$

$97.9<0.001$

$\begin{array}{ll}83.4 & 0.12\end{array}$

$11(10-12) \quad 0.0041$

Data are presented as \% of pulmonary rehabilitation programmes or median (interquartile range), unless otherwise stated. COPD: chronic obstructive pulmonary disease; ECT: energy conservation techniques; ADL: activities of daily life; PLB: pursed lips breathing. ${ }^{\#}$ : sum of proportions exceeds $100 \%$ as respondents were allowed or asked to choose more than one option; ${ }^{*}$ : pulmonary rehabilitation team members with a prevalence of $\geqslant 10 \%$ in one or both continents; ${ }^{+}: 50 \%$ of the programmes enrolled 76 to 105 patients (Europe) or 46 to 75 patients (North America) or more in $2011 ;{ }^{\S}$ : always including interstitial lung disease and sometimes including thoracic wall diseases; ${ }^{f}$ :weeks $\times$ sessions $\times$ time per session. 

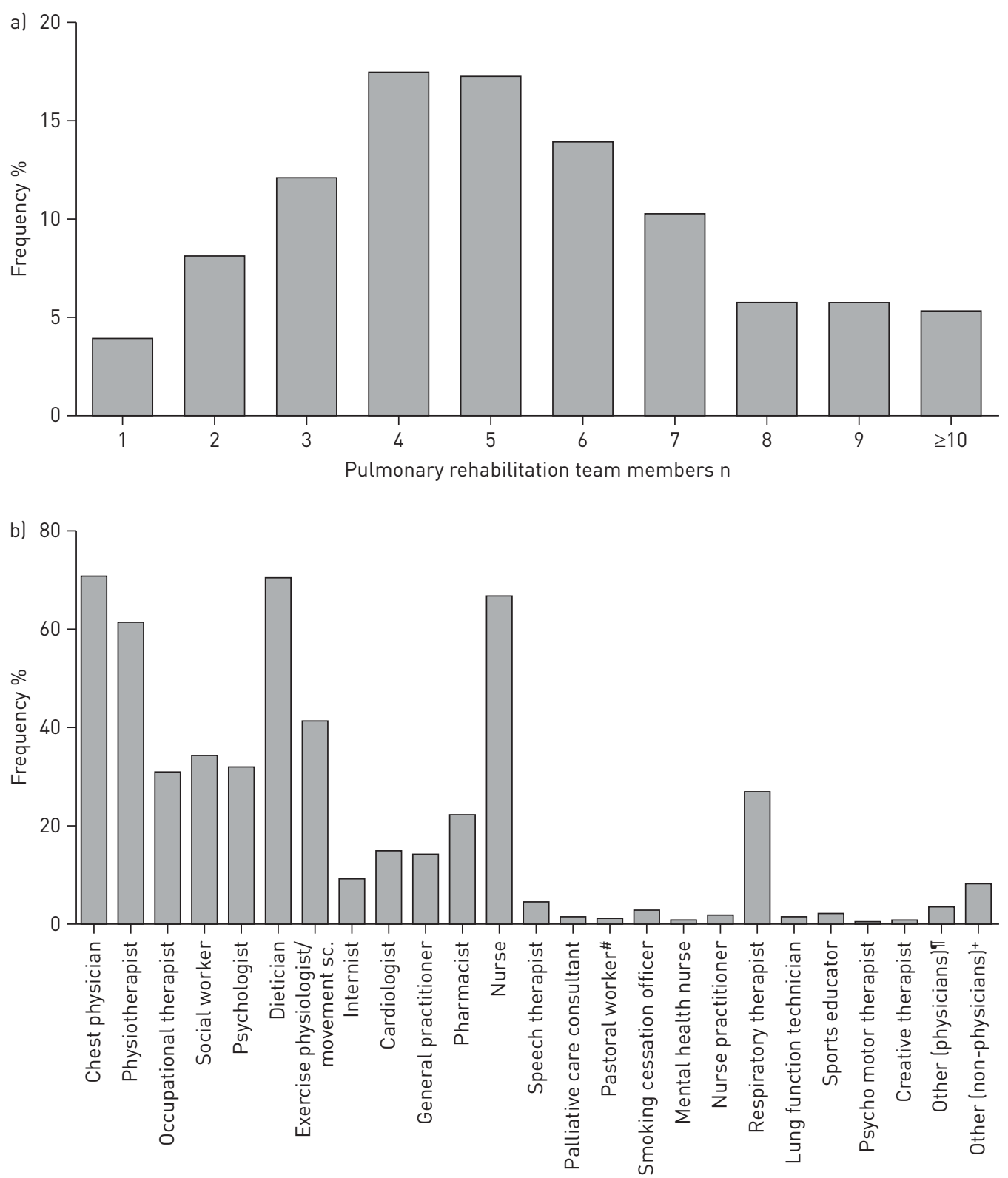

FIGURE 1 a) Number of pulmonary rehabilitation team members. b) Frequency of healthcare professionals. Movement sc.: movement scientist. ${ }^{\#}$ : includes, but not limited to, chaplain, parish nurse and pastoral worker; ": includes, but not limited to, geriatrician, neurologist, physiatrist, rheumatologist, surgeon and endocrinologist; ${ }^{+}$: includes, but not limited to, citizens advisor, volunteers, welfare rights advisor, council development worker, lung foundation support representative, dental hygienist, diabetes educators and alternative medicine practitioner.

\section{Most important outcomes}

Quality of life (82.1\% of the respondents), 6-min walk test (45.8\%) and dyspnoea (41.4\%) were identified as the three most important outcomes of pulmonary rehabilitation (fig. 5). Quality of life remained one of the three most important outcomes after stratification for Europe or North America (table 1). Programmes in Europe reported on dyspnoea and physical activity more often than in North America. North American programmes reported outcomes on the 6-min walk test, depression and smoking cessation more frequently than European programmes (table 1), although neither continent reported on depression and smoking cessation frequently.

\section{Interventions}

The median (IQR) number of types of interventions within the pulmonary rehabilitation programmes was 11 (9-12). The most common interventions were education $(94.4 \%)$, stationary cycling $(92.6 \%)$, breathing 


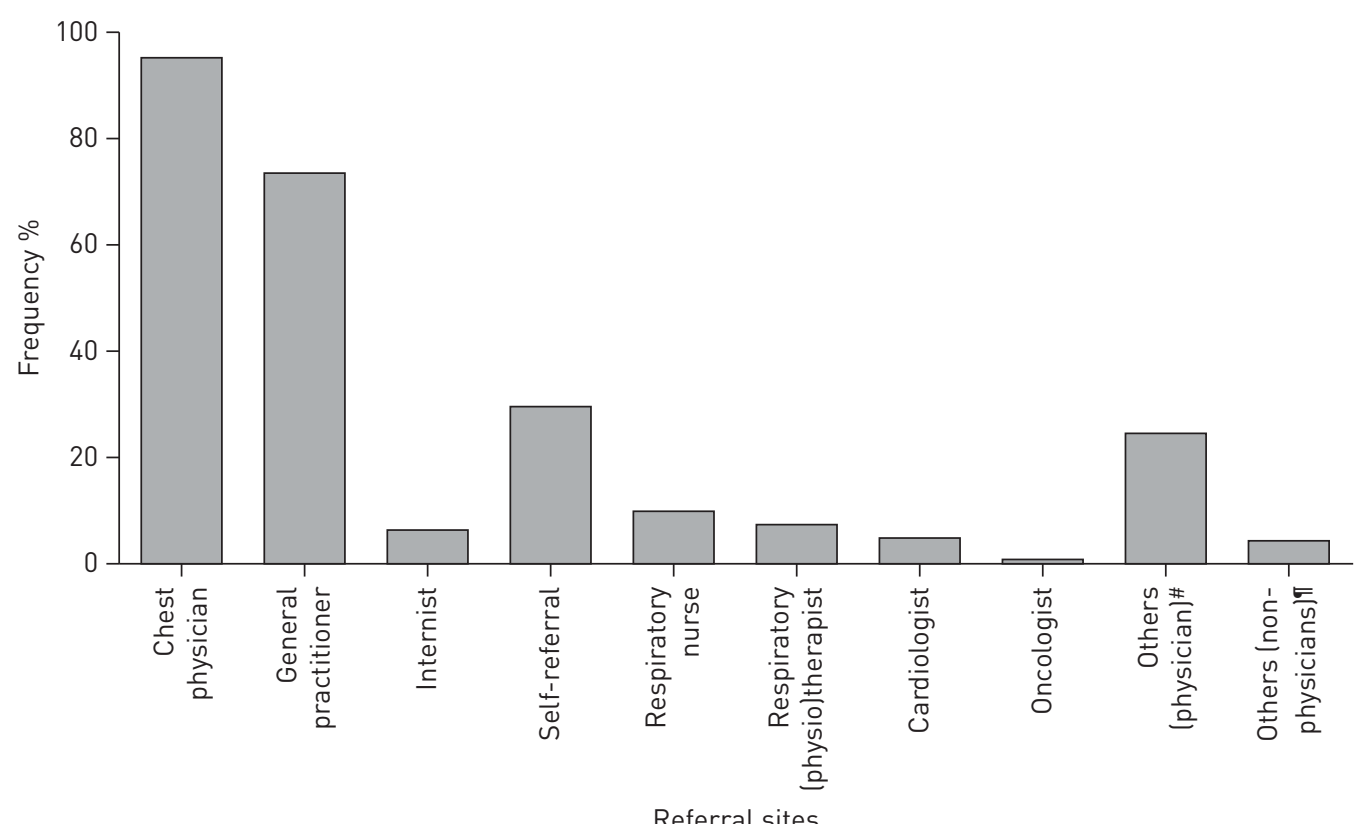

FIGURE 2 Pulmonary rehabilitation programme referral sources. ": includes, but not limited to, physiatrist, surgeon, "medical staff", "hospitalist", geriatrician and paediatrician; ": includes, but not limited to, waiting list from other pulmonary rehabilitation programme, community staff, occupational therapy, personal trainer, lung foundation and nutritionist.

exercises including pursed lips breathing (91.6\%) and nutritional support (84.4\%) (fig. 6). Outdoor walking and neuromuscular electrical stimulation were more commonly reported by programmes in Europe, while treadmill walking, stationary cycling, resistance training with handheld weights, education, training in activities of daily living, self-management, nutritional support, breathing exercises and psychosocial support were more commonly reported in North America (table 1). However, these differences were not great. For example, $89.4 \%$ of programmes in Europe reported on stationary cycling versus $97.3 \%$ in North America (table 1).

\section{Discussion}

This is the first global survey on content and organisational aspects of pulmonary rehabilitation. It was completed by representatives of 430 centres from 40 countries and, as expected, the findings clearly show key similarities, as well as substantial differences, between pulmonary rehabilitation programmes across continents. The observed differences make (inter)national benchmarking difficult, and suggest caution in generalisation of research findings between such different pulmonary rehabilitation programmes.

\section{Differences in content and organisational aspects}

Pulmonary rehabilitation for individuals with chronic respiratory disease is recommended by national and international guidelines and statements $[1,20]$. The current findings show differences in each of the categories surveyed: the setting, the case mix of individuals, the composition of the team, completion rates, methods of referral, and types of reimbursement. These findings corroborate previous reports performed at a national level $[11,14-17,21]$.

An encouraging finding was the use of health-related quality of life as the primary outcome of programmes, irrespective of jurisdiction. This reflects an evidence-based approach to evaluating the most important endpoint of pulmonary rehabilitation. Unfortunately, the current survey did not allow us to identify which questionnaires are used to assess health-related quality of life in daily clinical practice. Nevertheless, multiple options are available [22]. Approximately one-third of programmes employed measures of selfmanagement as part of their primary outcomes which, given the growing interest in self-management, especially around medication use and recognition of COPD exacerbations, is encouraging. Similarly, an awareness of the impact of pulmonary rehabilitation on activities of daily living was reflected by a number of programmes in both major jurisdictions using it as an outcome. Just over half of the programmes in North America utilise the 6-min walk test as a field measure of exercise capacity. Whereas this test is far less common in other parts of the world, the survey did not enable us to evaluate the frequency of alternative 

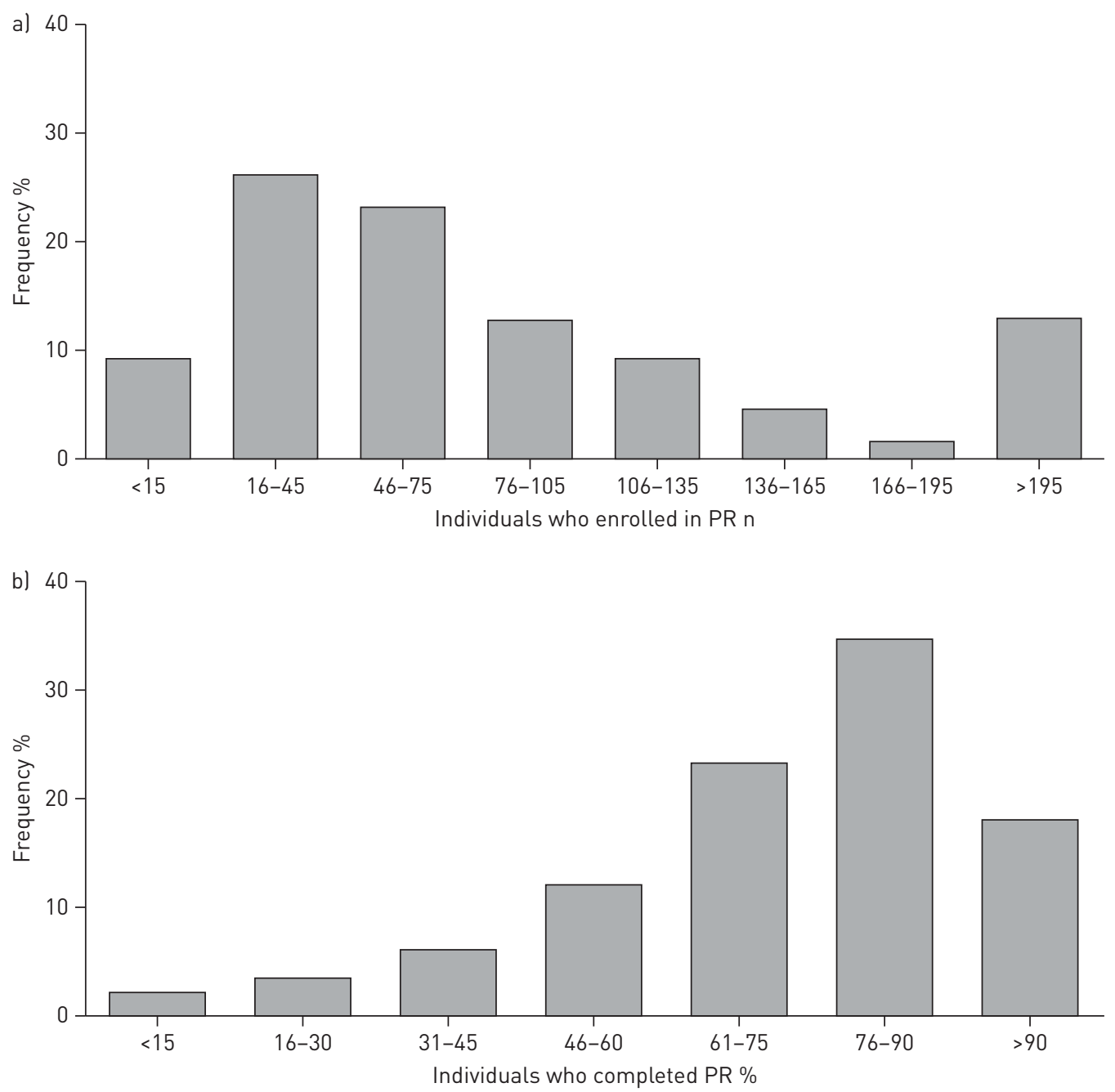

FIGURE 3 a) Estimated number of individuals who enrolled in each pulmonary rehabilitation (PR) programme in 2011. b) Estimated proportion of individuals who completed a PR programme in 2011.

field tests such as the shuttle walk test, which has become more popular in the past decade [23]. A small percentage of respondents included lung function as one of the three most important outcome measures for pulmonary rehabilitation. Such changes, as might occur, almost certainly reflect optimisation of pharmacotherapy, which should occur prior to programme enrolment. Disappointingly, few programmes identified the important psychological secondary impairments of anxiety and depression as part of their primary outcome measures. Given the prevalence of these symptoms and the positive impact that exercise has on them, increasing their use as outcome measures would be helpful $[24,25]$. The limited use of healthcare resource utilisation $(0.7 \%)$, despite clear evidence of the impact of rehabilitation on this outcome, was also unfortunate as the latter provides powerful reasons in support of programme funding related to significant reductions in healthcare costs among individuals with COPD [26]. Longitudinal data collection of healthcare resource utilisation, currently most likely to occur primarily in academic programmes, should be encouraged for community- and home-based environments.

Patient satisfaction and programmes safety, efficiency and accessibility were not identified as one of the top three most important outcomes of pulmonary rehabilitation. Even though this may not be recognised to be as important as performance metrics, it will provide detailed information about the pulmonary rehabilitation process. Moreover, healthcare facilities are increasingly being required to demonstrate these metrics as a condition of ongoing funding.

\section{Case mix}

The case mix of individuals with chronic respiratory diseases referred for pulmonary rehabilitation is highly variable, but indicates that the majority of the pulmonary rehabilitation programmes, in addition to 

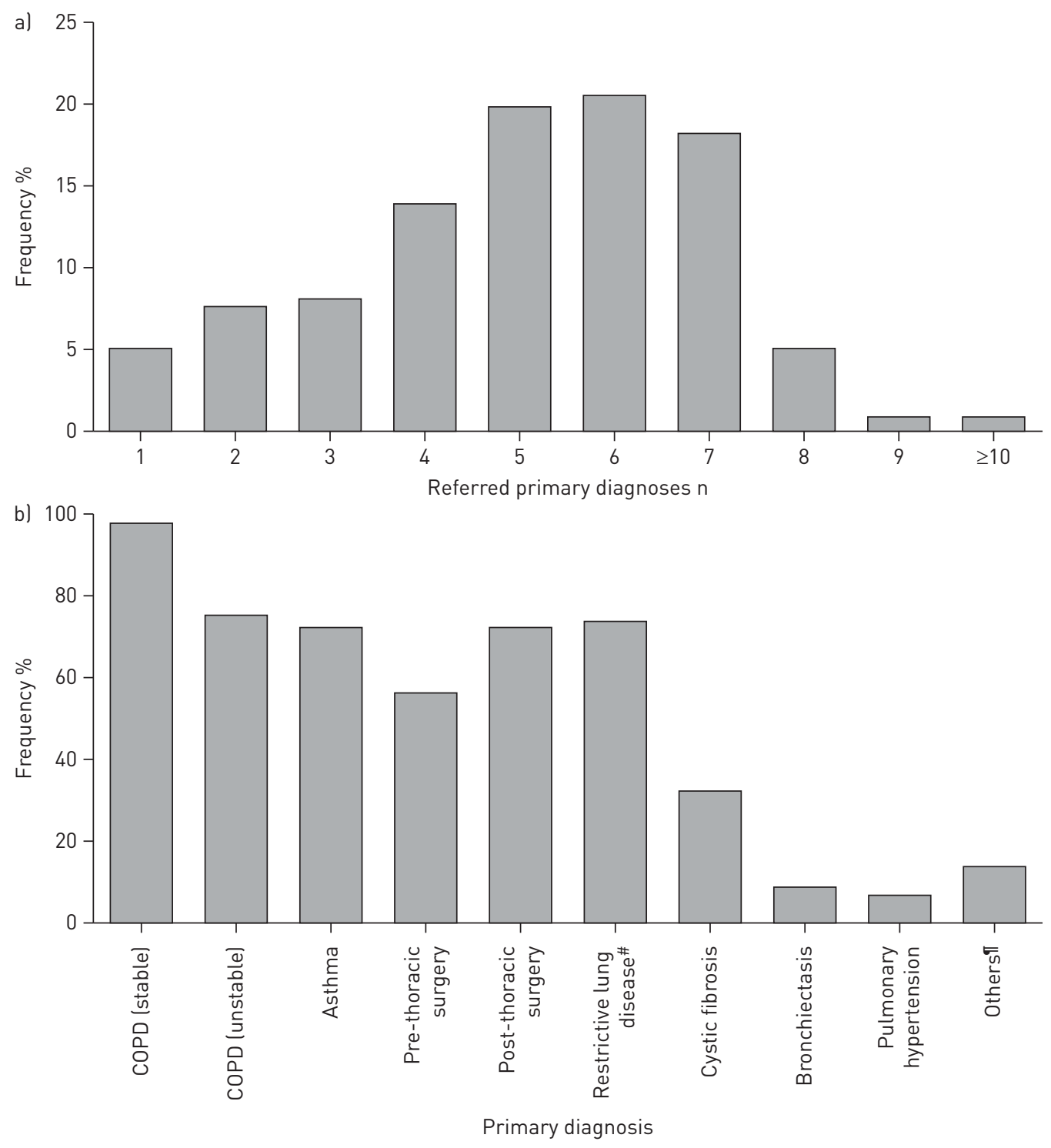

FIGURE 4 a) Number of primary diagnoses in each pulmonary rehabilitation programme. b) Frequency of primary diagnoses. ": includes, but not limited to, interstitial lung diseases such as sarcoidosis and idiopathic pulmonary fibrosis, and chest wall disease such as kyphoscoliosis; ${ }^{\natural}$ : includes, but not limited to, neuromuscular disease, diaphragm paresis, hyperventilation, chronic heart failure, post-intensive care unit, obesity, pre-bariatric surgery and post-bone marrow transplantation.

enrolling stable COPD patients, will also enrol individuals during or directly after a COPD exacerbation. Many also enrol individuals with a chronic respiratory disease other than COPD in keeping with the evolving scope of pulmonary rehabilitation [5, 9, 27-29]. Obviously, the primary respiratory diagnosis (i.e. COPD) does not provide healthcare professionals with enough phenotypic information to come up with a patient-tailored pulmonary rehabilitation programme. Therefore, a broad initial assessment is inevitable [30].

\section{Pulmonary rehabilitation teams}

An interdisciplinary pulmonary rehabilitation team should include physicians and skill sets associated with other healthcare professionals, such as physiotherapists, respiratory therapists, nurses, psychologists, behavioural specialists, exercise physiologists, nutritionists, occupational therapists and social workers [1]. The number and type of healthcare professionals varied amongst pulmonary rehabilitation programmes (fig. $1 \mathrm{a}$ and $1 \mathrm{~b}$ ), with physical therapists being especially popular in Europe and respiratory therapists (who do not exist in Europe) dominating North American programmes. In keeping with the interdisciplinary nature of pulmonary rehabilitation, available skill sets rather than specific healthcare professionals extends the programme capability. It seems that some tasks are interchangeable with other healthcare professionals 


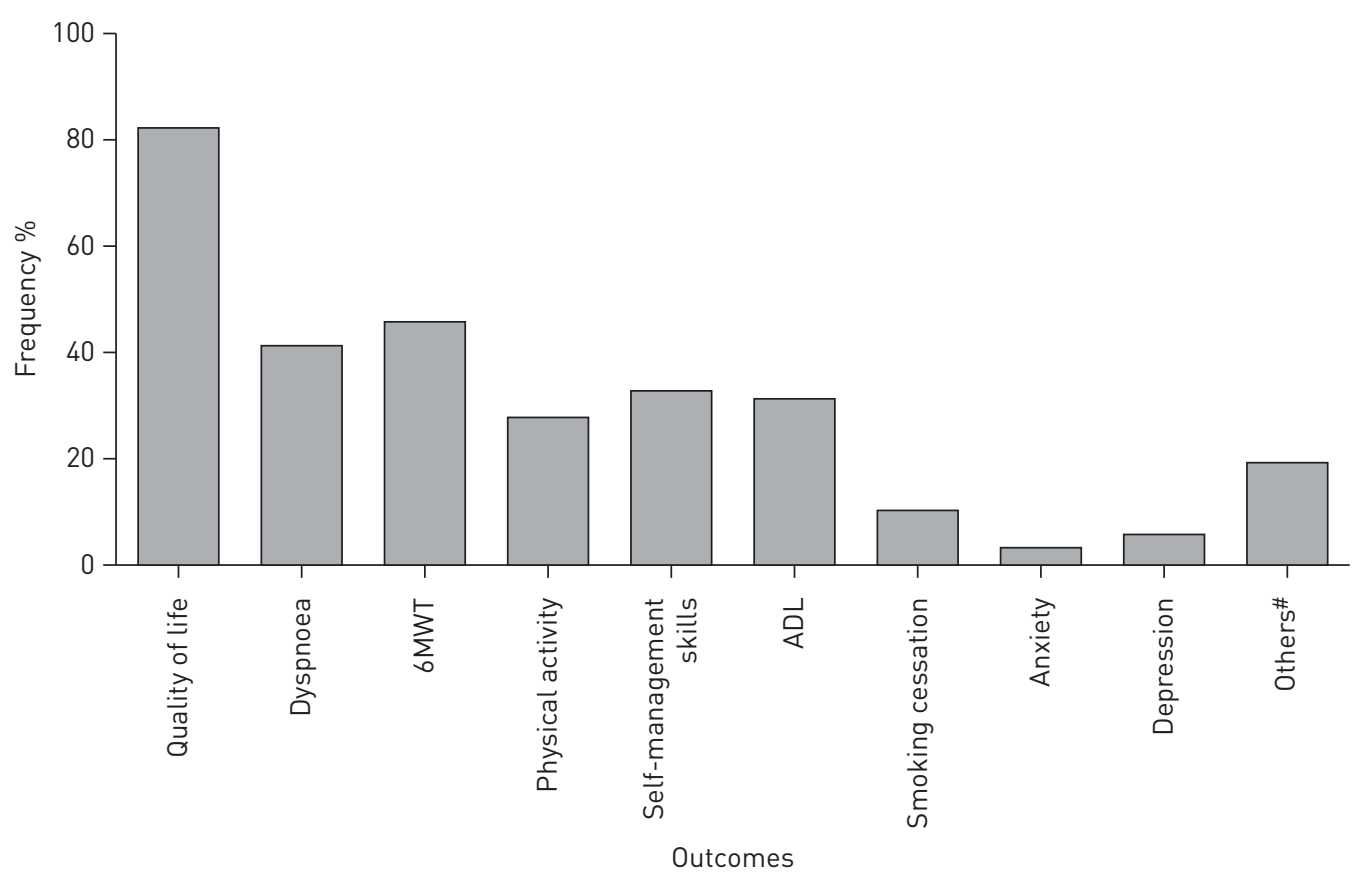

FIGURE 5 Frequency of the most important outcomes in the pulmonary rehabilitation programme. 6MWT: 6-min walk test; ADL: activities of daily living. "\#: includes, but not limited to, lung function, body composition, drug use, shuttle walk test, lower-limb muscle strength, upper-limb muscle strength and patient goals.

in daily clinical practice. For example, energy conservation techniques and/or activities of daily living training were available in $79.8 \%$ of the programmes, while an occupational therapist was only available in $30.9 \%$ of programmes.

17 "pulmonary rehabilitation teams" consisted of only one type of healthcare professional. This is somewhat surprising, as individuals with chronic respiratory disease can be very complex and need an integrated approach. Indeed, individuals with a chronic respiratory disease may present with multiple extrapulmonary features and comorbidities, such as symptoms of anxiety and depression, body composition abnormalities, cognitive dysfunction, lower-limb muscle weakness, cardiovascular disease and problematic activities of daily life [31-35]. These extrapulmonary features and comorbidities as well as poor self-management skills also need to be addressed during a comprehensive pulmonary rehabilitation programme $[36,37]$. Indeed, many programmes offered speciality components addressing extra-pulmonary features and/or comorbidities, e.g. $84.4 \%$ nutritional support, $79.8 \%$ energy conservation techniques and/or activities of daily living training, and $72.3 \%$ psychosocial support. Moreover, $80 \%$ of the programmes offered self-management training, which is believed to be necessary to achieve a meaningful and sustainable behaviour change [1].

\section{International benchmarking}

The observed differences in content and organisational aspects of pulmonary rehabilitation make benchmarking difficult among the various jurisdictions. Major differences in content and organisation were identified between programmes located in Europe and North America. It is unlikely that these differences are reported in clinical studies of rehabilitation and it is unknown as to whether variables such as team composition, skill mix, location and sources of payment have a direct impact on primary outcomes. Indeed, it is not clear whether extrapolating evidence from randomised controlled trials designed around a particular model of pulmonary rehabilitation may be generalised to other models.

The development of uniform performance and process metrics will enable more meaningful comparisons among programmes in different jurisdictions. It will also allow quality control to ensure appropriate standards for pulmonary rehabilitation. As a result, international scientific groups, such as the ERS Pulmonary Rehabilitation and Chronic Care and Physiotherapists groups, the AACVPR and the ATS Pulmonary Rehabilitation Assembly would greatly benefit from discussing future steps on how to compare the processes and results of other pulmonary rehabilitation programmes to one's own results. An international benchmarking approach could even be used to set aspirational targets. Obviously, the number of performance and process metrics need to be limited to retain manageability [38]. However, a 


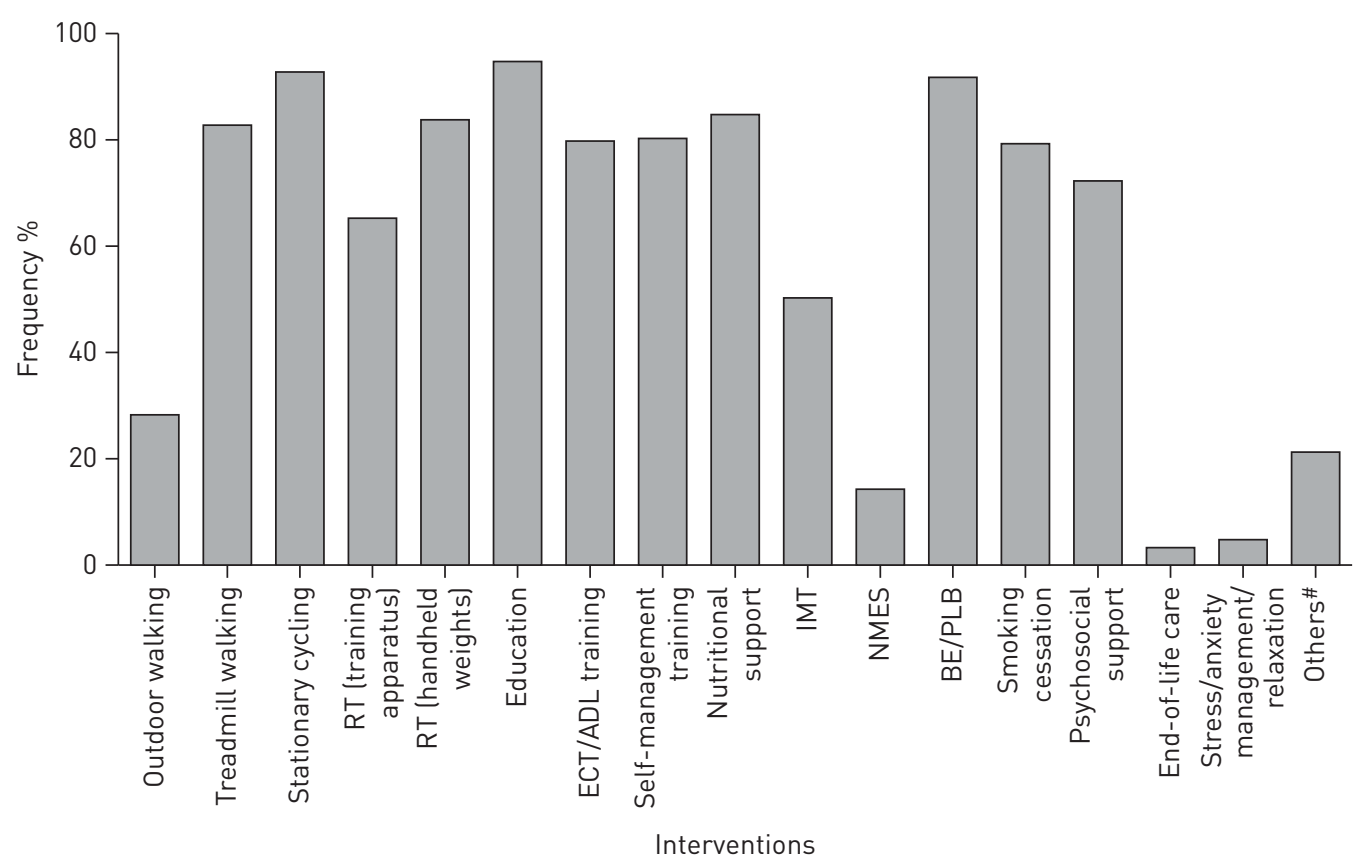

FIGURE 6 Frequency of the types of interventions in the pulmonary rehabilitation programme. RT: resistance training; ECT: energy conservation techniques; ADL: activities of daily life; IMT: inspiratory muscle training; NMES: neuromuscular electrical stimulation; BE: breathing exercise; PLB: pursed lips breathing; \#: includes, but not limited to, other types of physical exercise training, goal setting, airway clearance techniques, water therapy, psychomotor therapy, enhanced art therapy, arm cranking and support group.

performance dashboard which produces reports easily using standardised metrics can generate insights between performance and process metrics that are currently lacking in the field of pulmonary rehabilitation.

\section{Methodological limitations}

Although the total number of pulmonary rehabilitation programmes present worldwide is unknown, it is undoubtedly substantially greater than 430. Therefore, we acknowledge that, since many pulmonary rehabilitation programmes did not share their details, our study results may be subject to selection bias. We believe some of the reasons behind non-response to our inquiry may have been that some healthcare professionals involved in pulmonary rehabilitation: were not proficient in English (especially in Asia, South America and Africa); were not aware of this survey; or were too busy to reply. Therefore, the current findings must be considered hypothesis-generating rather than definitive. Based on the current findings, it is not possible to ascertain whether regional differences in pulmonary rehabilitation programmes are due to genuine differences in approach to pulmonary rehabilitation, health inequalities, differences in local healthcare systems, or other unrecognised factors. Nevertheless, this survey can be seen as the first step towards possible future uniformity concerning performance and process metrics in pulmonary rehabilitation.

In conclusion, large differences exist in content and organisational aspects among pulmonary rehabilitation programmes worldwide. The current findings stress the importance of the future development of process and performance metrics to monitor pulmonary rehabilitation programmes, to be able to start international benchmarking, and to provide recommendations for international standards based on evidence and best practice. Differences in content and organisational aspects of pulmonary rehabilitation programmes suggest caution in generalisation of research findings.

\section{Acknowledgements}

The authors' affiliations are as follows. M.A. Spruit: Dept of Research and Education, Centre of Expertise for Chronic Organ Failure (CIRO+), Horn, the Netherlands, and Rehabilitation Research Center (REVAL), BIOMED, Faculty of Medicine and Life Sciences, Hasselt University, Diepenbeek, Belgium. F. Pitta: Laboratório de Pesquisa em Fisioterapia Pulmonar, Departamento de Fisioterapia, Universidade Estadual de Londrina, Londrina, Brazil. C. Garvey: Pulmonary and Cardiac Rehabilitation Dept, Seton Medical Center, Daly City, CA, USA. R.L. ZuWallack: Dept of Pulmonary and Critical Care Medicine, St. Francis Hospital Medical Center, Hartford, CT, USA. C.M. Roberts: Barts and The London School of Medicine and Dentistry, Queen Mary University of London, London, UK. E.G. Collins: Research and Development, Edward Hines, Jr. VA Hospital, Hines, IL, and Department of Biobehavioral Health Science, University of Illinois at Chicago, Chicago, IL, USA. R. Goldstein: Dept of Respiratory Medicine, University of Toronto, Toronto, ON, Canada. R. McNamara: Depts of Respiratory Medicine and Physiotherapy, Prince of Wales Hospital, Sydney, Australia. 
P. Surpas: Centre Médical de Bayère, Charnay, France. K. Atsuyoshi: Dept of Rehabilitation, Akita City General Hospital, Akita, Japan. J-L. López-Campos: Unidad Medico-Quirugica de Enfermedades Respiratorias, Instituto de Biomedicina de Sevilla (IBiS), Hospital Universitario Virgen del Rocío, Seville, and CIBER de Enfermedades Respiratorias (CIBERES), Instituto de Salud Carlos III, Madrid, Spain. I. Vogiatzis: Pulmonary Rehabilitation Unit, 1st Dept of Respiratory Medicine and Dept of Physical Education and Sport Sciences, National and Kapodistrian University of Athens, Athens, Greece. J.E.A. Williams: Centre for Exercise and Rehabilitation Science, Glenfield Hospital, University Hospitals of Leicester NHS Trust, Leicester, UK. S. Lareau: College of Nursing, University of Colorado, Denver, CO, USA. D. Brooks: Dept of Physical Therapy, Faculty of Medicine, University of Toronto, Toronto, ON, Canada. T. Troosters: Dept of Rehabilitation Sciences, Katholieke Universiteit Leuven, and Dept of Respiratory Rehabilitation and Respiratory Division, University Hospital Leuven, Leuven, Belgium. S.J. Singh: Centre for Exercise and Rehabilitation Science, Glenfield Hospital, University Hospitals of Leicester NHS Trust, Leicester, UK. S. Hartl: Ludwig Boltzmann Institute of COPD and Respiratory Epidemiology, Vienna, Austria. E.M. Clini: Dept of Medical and Surgical Sciences, University of Modena and Ospedale Villa Pineta, Modena, Italy. E.F.M. Wouters: Dept of Research and Education, Centre of Expertise for Chronic Organ Failure (CIRO+), Horn, and Dept of Respiratory Medicine, Maastricht University Medical Center+, the Netherlands.

We are grateful to all our pulmonary rehabilitation colleagues who completed and returned the surveys and to I. Timmermans (Centre of Expertise for Chronic Organ Failure (CIRO+), Horn, the Netherlands) for inserting all the surveys into a database.

\section{References}

1 Spruit MA, Singh SJ, Garvey C, et al. An official American Thoracic Society/European Respiratory Society statement: key concepts and advances in pulmonary rehabilitation. Am J Respir Crit Care Med 2013; 188: e13-e64. Hill K, Vogiatzis I, Burtin C. The importance of components of pulmonary rehabilitation, other than exercise training, in COPD. Eur Respir Rev 2013; 22: 405-413.

3 Blanco I, Santos S, Gea J, et al. Sildenafil to improve respiratory rehabilitation outcomes in COPD: a controlled trial. Eur Respir J 2013; 42: 982-992.

4 Gouzi F, Préfaut C, Abdellaoui A, et al. Blunted muscle angiogenic training-response in COPD patients versus sedentary controls. Eur Respir J 2013; 41: 806-814.

5 Huppmann P, Sczepanski B, Boensch $\mathrm{M}$, et al. Effects of inpatient pulmonary rehabilitation in patients with interstitial lung disease. Eur Respir J 2013; 42: 444-453.

6 McNamara RJ, McKeough ZJ, McKenzie DK, et al. Water-based exercise in COPD with physical comorbidities: a randomised controlled trial. Eur Respir J 2013; 41: 1284-1291.

7 Paddison JS, Effing TW, Quinn S, et al. Fatigue in COPD: association with functional status and hospitalisations. Eur Respir J 2013; 41: 565-570.

8 Gloeckl R, Marinov B, Pitta F. Practical recommendations for exercise training in patients with COPD. Eur Respir Rev 2013; 22: 178-186.

9 Holland AE, Wadell K, Spruit MA. How to adapt the pulmonary rehabilitation programme to patients with chronic respiratory disease other than COPD. Eur Respir Rev 2013; 22: 577-586.

10 Wagg K. Unravelling self-management for COPD: what next? Chron Respir Dis 2012; 9: 5-7.

11 Brooks D, Sottana R, Bell B, et al. Characterization of pulmonary rehabilitation programs in Canada in 2005. Can Respir J 2007; 14: 87-92.

12 Levack WM, Weatherall M, Reeve JC, et al. Uptake of pulmonary rehabilitation in New Zealand by people with chronic obstructive pulmonary disease in 2009. N Z Med J 2012; 125: 23-33.

13 Yohannes A, Stone R, Lowe D, et al. Pulmonary rehabilitation in the United Kingdom. Chron Respir Dis 2011; 8: 193-199.

14 Wadell K, Janaudis Ferreira T, Arne M, et al. Hospital-based pulmonary rehabilitation in patients with COPD in Sweden - a national survey. Respir Med 2013; 107: 1195-1200.

15 Johnston CL, Maxwell LJ, Alison JA. Pulmonary rehabilitation in Australia: a national survey. Physiotherapy 2011; 97: 284-290.

16 Brooks D, Lacasse Y, Goldstein RS. Pulmonary rehabilitation programs in Canada: national survey. Can Respir J 1999; 6: 55-63.

17 Yohannes AM, Connolly MJ. Pulmonary rehabilitation programmes in the UK: a national representative survey. Clin Rehabil 2004; 18: 444-449.

18 Lacasse Y, Martin S, Lasserson TJ, et al. Meta-analysis of respiratory rehabilitation in chronic obstructive pulmonary disease. A Cochrane systematic review. Eura Medicophys 2007; 43: 475-485.

19 Garvey C, Spruit MA, Hill K, et al. International COPD Coalition Column: pulmonary rehabilitation - reaching out to our international community. J Thorac Dis 2013; 5: 343-348.

20 Ries AL, Bauldoff GS, Carlin BW, et al. Pulmonary rehabilitation: joint ACCP/AACVPR evidence-based clinical practice guidelines. Chest 2007; 131: Suppl. 5, 4S-42S.

21 Pozo-Rodríguez F, López-Campos JL, Alvarez-Martínez CJ, et al. Clinical audit of COPD patients requiring hospital admissions in Spain: AUDIPOC study. PLoS One 2012; 7: e42156.

22 Dodd JW, Marns PL, Clark AL, et al. The COPD Assessment Test (CAT): short- and medium-term response to pulmonary rehabilitation. COPD 2012; 9: 390-394.

23 Singh SJ, Jones PW, Evans R, et al. Minimum clinically important improvement for the incremental shuttle walking test. Thorax 2008; 63: 775-777.

24 Harrison SL, Greening NJ, Williams JE, et al. Have we underestimated the efficacy of pulmonary rehabilitation in improving mood? Respir Med 2012; 106: 838-844.

25 Trappenburg JC, Troosters T, Spruit MA, et al. Psychosocial conditions do not affect short-term outcome of multidisciplinary rehabilitation in chronic obstructive pulmonary disease. Arch Phys Med Rehabil 2005; 86: 1788-1792.

26 Raskin J, Spiegler P, McCusker C, et al. The effect of pulmonary rehabilitation on healthcare utilization in chronic obstructive pulmonary disease: the Northeast Pulmonary Rehabilitation Consortium. J Cardiopulm Rehabil 2006; 26: 231-236. 
27 Spruit MA, Janssen DJ, Franssen FM, et al. Rehabilitation and palliative care in lung fibrosis. Respirology 2009; 14: 781-787.

28 Spruit MA, Janssen PP, Willemsen SC, et al. Exercise capacity before and after an 8-week multidisciplinary inpatient rehabilitation program in lung cancer patients: a pilot study. Lung Cancer 2006; 52: 257-260.

29 Ambrosino N, Venturelli E, Vagheggini G, et al. Rehabilitation, weaning and physical therapy strategies in chronic critically ill patients. Eur Respir J 2012; 39: 487-492.

30 Spruit MA, Vanderhoven-Augustin I, Janssen PP, et al. Integration of pulmonary rehabilitation in COPD. Lancet 2008; 371: 12-13.

31 Graat-Verboom L, Wouters EF, Smeenk FW, et al. Current status of research on osteoporosis in COPD: a systematic review. Eur Respir J 2009; 34: 209-218.

32 Janssen DJ, Spruit MA, Leue C, et al. Symptoms of anxiety and depression in COPD patients entering pulmonary rehabilitation. Chron Respir Dis 2010; 7: 147-157.

33 Vanfleteren LE, Spruit MA, Groenen M, et al. Clusters of comorbidities based on validated objective measurements and systemic inflammation in patients with chronic obstructive pulmonary disease. Am J Respir Crit Care Med 2013; 187: 728-735.

34 Rutten EP, Breyer MK, Spruit MA, et al. Abdominal fat mass contributes to the systemic inflammation in chronic obstructive pulmonary disease. Clin Nutr 2010; 29: 756-760.

35 Sillen MJ, Franssen FM, Delbressine JM, et al. Heterogeneity in clinical characteristics and co-morbidities in dyspneic individuals with COPD GOLD D: findings of the DICES trial. Respir Med 2013; 107: 1186-1194.

36 Crisafulli E, Costi S, Luppi F, et al. Role of comorbidities in a cohort of patients with COPD undergoing pulmonary rehabilitation. Thorax 2008; 63: 487-492.

37 Crisafulli E, Gorgone P, Vagaggini B, et al. Efficacy of standard rehabilitation in COPD outpatients with comorbidities. Eur Respir J 2010; 36: 1042-1048.

38 Ettorchi-Tardy A, Levif M, Michel P. Benchmarking: a method for continuous quality improvement in health. Healthc Policy 2012; 7: e101-e119. 